\title{
Thromboinflammation in COVID-19: The Clot Thickens
}

\author{
Raayma Iffah ${ }^{1}$ and Felicity Gavins ${ }^{1}$ \\ ${ }^{1}$ Brunel University
}

April 18, 2021

\begin{abstract}
Since the start of the novel coronavirus SARS-Cov-2 pandemic, a disease that has become one of the world's greatest global health challenges, the role of the immune system has been at the forefront of scientific studies. The pathophysiology of COVID-19 is complex, which is evident by those at higher risk for poor outcome. Multiple systems contribute to thrombosis and inflammation seen in COVID-19 patients, including neutrophil dysfunction, platelet activation, endothelial cell activation. Understanding how the immune system functions in different patient cohorts (particularly given recent emerging events with the Oxford/AstraZeneca vaccine) is vital to understanding the pathophysiology of this devastating disease and for subsequent development of novel therapeutic targets and expedite possible drug repurposing strategies that could benefit society on a global scale.
\end{abstract}

Thromboinflammation in COVID-19: The Clot Thickens

Raayma Iffah ${ }^{1}$ and Felicity N.E. Gavins ${ }^{1 *}$

${ }^{1}$ Department of Life Sciences, The Centre for Inflammation Research and Translational Medicine (CIRTM), Brunel University London, Uxbridge, Middlesex, UB8 3PH, UK.

\section{*Corresponding author:}

Felicity N. E. Gavins

Department of Life Sciences

Brunel University London

Kingston Lane

London

Uxbridge

UB8 3PH

Tel: +44 (0) 1895267151

E-mail: felicity.gavins@brunel.ac.uk

Word count: 1973

Data Availability: No data have been shared.

Abstract 
Since the start of the coronavirus SARS-Cov-2 pandemic, a disease that has become one of the world's greatest global health challenges, the role of the immune system has been at the forefront of scientific studies. The pathophysiology of COVID-19 is complex, which is evident in those at higher risk for poor outcome. Multiple systems contribute to thrombosis and inflammation seen in COVID-19 patients, including neutrophil and platelet activation, and endothelial dysfunction. Understanding how the immune system functions in different patient cohorts (particularly given recent emerging events with the Oxford/AstraZeneca vaccine) is vital to understanding the pathophysiology of this devastating disease and for the subsequent development of novel therapeutic targets and to facilitate possible drug repurposing strategies that could benefit society on a global scale.

Keywords: Thrombosis, inflammation, thromboinflammation, neutrophils, platelets, resolution pharmacology

\section{Introduction}

Severe Acute Respiratory Syndrome Coronavirus 2 (SARS-CoV-2) is the aetiological agent of coronavirus disease 2019 (COVID-19), a disease that has had global impact, with case rates surging to over 3,000 deaths a day across Europe alone. Moreover, since the initial reported emergence in December 2019 in the Hubei province of Wuhan, China, and the World Health Organisation (WHO) declaring the COVID-19 outbreak a pandemic (11 March 2020), 128 million confirmed cases of COVID-19 have been reported globally, including 2.8 million deaths. As of this year (2021) there have been profound breakthroughs such as the approval and administration of vaccines from Pfizer-BioNTech, Moderna, and Oxford/AstraZeneca, with additional vaccines from Valneva, CureVac Novavax and Johnson \& Johnson currently under assessment by the Medicines and Healthcare products Regulator Agency (MHRA), with others in the pipeline (e.g. GlaxoSmithKline/Sanofi Pasteur's vaccine which may be available by the final quarter of 2021).

\subsection{Coronaviruses $(\mathrm{CoV})$}

$\mathrm{CoV}$, of which there are four genera (alpha, beta, gamma, and delta), are a family of enveloped, positivesense, single-stranded, and highly diverse RNA viruses.(Zhu et al. 2020) Three beta coronaviruses have been identified to cause severe respiratory illness in humans including SARS-CoV, and MERS-CoV (Middle Eastern respiratory syndrome CoV) and SARS-CoV-2.(McFadyen, Stevens, and Peter 2020) Although bats have been suggested to be the primary origin of SARS-CoV and SARS-CoV-2, the precise origin and mechanism by how SARS-CoV-2 manifested in humans is still unclear, with other possible hosts (such as the Malayan pangolins [Manis javanica ]), under investigation.(Lam et al. 2020)

SARS-CoV-2 is a single-stranded RNA virus and entry into host cells initiates with their transmembrane spike (S) glycoproteins (GP) encompassing S1 and S2 subunits. The S1 unit binds to host cell receptors, while the S2 subunit aids in viral and host cell fusion.(Walls et al. 2020) Like SARS-CoV, SARS-CoV2 binds via the human angiotensin-converting enzyme 2 (ACE-2) receptor expressed on the endothelial surface.(Davidson, Wysocki, and Batlle 2020; Hoffmann et al. 2020) In comparison to SARS-CoV, SARSCoV-2 has different amino acid residues within the receptor-binding domain (RBD) of the $\mathrm{S}$ protein and a polybasic furin cleavage site at the junction of S1 and S2. In addition, transmissibility and pathogenicity of SARS-CoV-2 is linked to cellular proteases such as furin and transmembrane protease serine 2 (TMPRSS2).(McFadyen, Stevens, and Peter 2020) Although the functionality of these specific features of SARS-CoV-2 has yet to be fully elucidated, they could provide key information to the pro-thrombotic phenotype associated with SARS-CoV-2 and why patients with this specific Co-V have higher thrombosis rates vs. SARS-CoV and MERS-CoV infections.

\subsection{Clinical manifestations and thrombo-inflammation}

Since the discovery of SARS-Cov-2 infection, a number of clinical manifestations such as: fever, dry cough, malaise, sore throat, chest pain, dyspnoea, nausea, diarrhoea and vomiting.(Tian et al. 2020) The spectrum of symptomatic infection ranges from mild to critical, although there remains uncertainty around the proportion 
of asymptomatic infections. Among hospitalised patients, the proportion of critical or fatal disease is higher, with many progressing to acute respiratory distress syndrome (ARDS), respiratory failure and eventually death.(Bost et al. 2021) Interestingly, the ARDS associated with COVID-19 patients differs from that caused by other infective or traumatic insults, with the "cytokine storm" (i.e. increased cytokines released from the blood, which is associated with disease severity) being only partially involved in COVID-19 patients.(Maier et al. 2020) Furthermore, interleukin (IL)-6 levels are 60-90-fold higher in ARDS patients compared to COVID19 patients, although the reasons for these differences are unknown. It is conceivable that SARS-CoV-2 takes over the host immune system, impairing antiviral immunity and triggering chronic inflammation by involving inflammatory cytokines.(Sinha, Matthay, and Calfee 2020; Bost et al. 2021) Certainly a hallmark of SARSCoV-2 is the release of not only IL-6, but other cytokines and chemokines including IL-1 $\beta$, IL-2, IL-6, IL-7, IL-8, IL-10, IL-17, TNF- $\alpha$, chemokine ligand-2 (CCL2), CCL3, interferon gamma-inducible protein, C-X-C motif chemokine ligand-10, and monocyte chemoattractant protein-1, all of which correlate with adverse clinical outcomes. The inflammatory effects of cytokines also activate vascular endothelial cells, disrupting endothelial function and integrity, which leads to increased platelet and leukocyte recruitment, resulting in a pro-inflammatory and pro-thrombotic state.(Connors and Levy 2020)

Autopsy reports from SARS-CoV-2 patients show multi-organ dysfunction, with highest viral titres in the lungs and immune cells, and the presence of endothelial inflammation and cell death.(Gu et al. 2005; Mazzulli et al. 2004) It is likely that hypoxia, also promotes a pro-thrombotic endothelial phenotype in SARS-CoV-2 via hypoxia-inducible transcription factor activation and endothelial tissue factor (TF) upregulation.

The scale of COVID-19 severity also increases with comorbidities such as hypertension, chronic kidney disease, obstructive sleep apnoea, asthma diabetes and obesity.(Tian et al. 2020) Patients with underlying cardiovascular disease are prone to increased severity of COVID-19 and a 5-fold increase in mortality.(Yang et al. 2020) Therefore, managing and controlling cardiovascular risk factors is a high priority.

COVID-19 is associated with a prothrombotic state, which can manifest as microvascular thrombosis, venous thromboembolism (VTE) or arterial thrombosis.(McFadyen, Stevens, and Peter 2020) The cause of this pro-thrombotic state may relate to the appreciated link between thrombosis and inflammation (termed 'thromboinflammation') in which thrombosis can amplify inflammation and systemic inflammation can beget thrombosis. Whilst efforts to understand the complex immunological landscape in COVID-19 are evolving, both platelet activation and platelet-neutrophil interactions play a crucial role in thromboinflammation.

\section{Role of Neutrophils in COVID-19}

Neutrophils are the immune system's first responders having crucial functions in immunity and repair. Upon activation, they produce pro-inflammatory cytokines (including IL-6, TNF- $\alpha$ and IL-1 $\beta$ ), generate reactive oxygen species (ROS), release hematopoietic serine proteases (neutrophil elastase, proteinase 3, and cathepsin G), microparticles and neutrophil extracellular traps (NETs). Neutrophils possess anti-microbial properties capable of not only killing both Gram-positive and Gram-negative bacteria, but they can also act as a double edged sword mediating tissue injury and perpetuating the inflammatory response.(Kolaczkowska and Kubes 2013) NETs, ROS and serine proteases can all independently, or collectively, upregulate thromboinflammatory processes.

In the context of COVID-19, neutrophilia signifies worse outcomes, with autopsy studies showing neutrophil infiltration in pulmonary capillaries, acute capillaries with fibrin deposition, extravasation of neutrophils in the alveolar space and neutrophil mucositis of the trachea.(Yao et al. 2020; Gu et al. 2005) Bost et al., revealed at least ten different neutrophil states present in blood and broncho-alveolar lavage of COVID-19 patients, with resting state phenotype mainly associated with mild patients and activated or immature phenotype associated with severe patients.(Bost et al. 2021) Their results suggested that COVID-19 is associated with a state of 'immune silence' (demonstrated by loss of neutrophil and monocyte immunosuppression and the replacement of lung memory CD8 $+\mathrm{T}$ cells by naïve $\mathrm{T}$ cells), correlating with severe clinical manifestation and outcome.(Bost et al. 2021) Diao et al., showed T-cells are dysfunctional with increased expression of exhaustion molecules related to heightened systemic inflammation, including IL-6 levels.(Diao et al. 2020) 
We have previously shown that IL-6 plays a major role in T-cell dependent Ang-II thromboinflammatory responses and in the activation and aggregation of platelets.(Senchenkova, Russell, et al. 2019) These findings support the hypothesis that drug discovery programmes based on T-cell dependent IL-6 signalling pathways may afford protection against thromboinflammation in COVID-19.

As observed with other pathological conditions (e.g. sickle cell disease),(Ansari et al. 2021) NET levels are increased in COVID-19 patients, with sera from COVID-19 patients being shown to trigger NET release from control neutrophils, and containing increased MPO-DNA complexes and citrullinated histone H3 levels which correlated with disease severity.(Veras et al. 2020; Zuo et al. 2020) These results suggest NETs may contribute to COVID-19 pathology and NET biomarkers may help to predict clinical worsening and VTE.

Neutrophil degranulation and NET formation exerts various intracellular danger-associated molecular patterns (DAMPs) activating pattern recognition receptors (PRRs) on nearby immune and non-immune cells releasing pro-inflammatory mediators.(Tomar et al. 2020) DAMPs activate properdin, factor B and C3, all components of the alternative pathway necessary to induce the complement cascade. Reports have shown increased activation of the complement system in severe COVID-19 patients.(Holter et al. 2020) Lung biopsies from COVID-19 patients have shown deposits of terminal complement components C5b-9, C4d, and mannose binding lectin (MBL)-associated serine protease (MASP)2, consistent with sustained, systemic activation of the complement pathways.(Magro et al. 2020) These findings highlight the therapeutic strategy of complement targeted therapies for COVID-19 mediated thrombosis.

The initial neutrophil response also leads to interactions with platelets via a variety of different mechanisms including Mac-1 (CD11b/CD18)/Glycoprotein Ib (CD42) and P-Selectin/P-selectin glycoprotein ligand-1 (PSGL-1), formation of fibrin cross-links (via Mac-1/fibrin interaction) and induction of extrinsic TF/Factor IIa pathway, generating thrombin.(De Meyer et al. 2016) TF-enriched NETs and a high neutrophil count are associated with increased disease severity and poor prognosis in COVID-19,(Skendros et al. 2020) amplifying the need for increased research regarding platelet-neutrophil interactions in thrombogenesis.

\section{Role of Platelets and in COVID-19}

Platelets are traditionally known for their role in haemostasis and formation of thrombi. However, they are increasingly being recognised as key effector cells in inflammation, influencing innate and adaptive immune responses.(Senchenkova, Ansari, et al. 2019) Migration of single platelets act as mechano-sensors to collect and bundle bacteria for neutrophil phagocytosis,(Gaertner et al. 2017) although their uncontrolled activation can result in pathogenic thrombosis. Platelets from COVID-19 patients show increased aggregation, adhesion and spreading on fibrinogen and collagen (via upregulation of the MAPK pathway) and increased activity of thromboxane $\mathrm{A}_{2}$ (marker of platelet activation).(Manne et al. 2020)

Thrombocytopenia in COVID-19 is common, with meta-analysis demonstrating a link with a 5 -fold increased risk of severe disease.(Lippi, Plebani, and Henry 2020) Thrombocytopenia has also been linked with issues of rare blood clotting events which now surround the Oxford/AstraZeneca vaccine (ongoing pharmacovigilance will determine outcome), with seven deaths being reported in the thirty cases being reported in the UK alone (at the time of writing this review, evidence is shifting towards a causal link between the vaccine and rare blood clots).

Elevated D-dimers, fibrinogen, and von Willebrand factor levels are also associated with a higher mortality rate. Autopsies of cardiac and pulmonary tissue from COVID-19 patients have shown the presence of megakaryocytes.(Rapkiewicz et al. 2020) A recent study by Manne et al., using platelet RNA sequencing demonstrated COVID-19 induced significant changes in platelet transcriptome and proteome, and platelet hyperreactivity, (Manne et al. 2020) which may contribute to COVID-19 pathophysiology by increasing platelet homotypic and heterotypic interactions. During infection, platelets became hyperactive indicated through increased surface P-selectin expression and increased formation of circulating platelet-neutrophil aggregates (PNAs) via binding with PSGL-1.(Manne et al. 2020) The generation of PNAs recruit neutrophils to damaged lung capillaries. Thrombotic events in COVID-19 may be attributed to platelets augmenting inflammation through the generation of NETs and procoagulant platelets, increased aggregates (e.g. PNAs), 
and the release of bioactive substances. A greater understanding of the role that neutrophils and platelets play in thromboinflammation and their involvement in the pathophysiology of COVID-19 is needed for drug discovery focussing on dampening thromboinflammatory processes.

\section{Therapeutic intervention thromboinflammation resolution}

Understanding neutrophil and platelet responses in the context thromboinflammation in COVID-19 has resulted in promising pre-clinical studies demonstrating that e.g. immune regulatory properties are being lost in patients with COVID-19, with the virus seemingly to 'suppress' or 'silence' the immune system.(Bost et al. 2021) Drugs that can 'stimulate' or 'reawaken' the immune system may be the way forward, as seen with dexamethasone.(Group et al. 2021; Bost et al. 2021) Other therapies may lie in targeting the cytokine storm with anti-inflammatory agents such as IL-6 and IL-1 $\beta$ antagonists.

Accumulating data linking inflammation and thrombosis supports the hypothesis that anti-inflammatory therapies may limit thrombosis and that anti-thrombotic therapies may reduce vascular inflammation.(Vital et al. 2016) Furthermore, there is a growing evidence of the importance of resolution biology in vascular inflammation to develop innovative approaches for the treatment of diseases, which may include COVID19. Inflammation resolution, is an active process involving specific endogenous mediators (e.g. Annexin A1 [AnxA1], aspirin triggered lipoxin $\mathrm{A}_{4}$ ), and pathways (e.g. formyl peptide receptor 2 [Fpr2/ALX] pathway).(Senchenkova, Ansari, et al. 2019) FPR2 agonists are being developed and currently tested in man. In the context of thromobinflammation and the possible insights for therapeutic strategies for COVID-19 treatments, we recently discovered targeting the AnxA1/Fpr2/ALX pathway promotes thromboinflammation resolution by altering both the platelet phenotype (from pro-pathogenic to regulatory)(Senchenkova, Ansari, et al. 2019) and the pathological neutrophil phenotype (from a pro-NETotic to pro-apoptotic).(Ansari et al. 2021)

\section{Conclusion}

COVID-19 is a devastating disease that has affected the UK and our global community in unprecedented ways. The pathophysiology is complex with multiple systems likely contributing to the pro-thrombotic state including inflammation, platelet and neutrophil activation, endothelial cell dysfunction, NETs and complement factors. However the mechanism(s) that drives COVID-19 associated thromboinflammation has not yet been fully elucidated and there is an urgent unmet clinical need to fully understand and characterise this disease. These discoveries will unearth ways to develop pharmacological strategies which may also focus on resolution biology.

\section{Acknowledgement}

FNEG acknowledges the support of The Royal Society Wolfson Foundation: RSWF $\backslash R 3 \backslash 183001$.

\section{Disclosures}

None.

\section{References}

Ansari, J., E. Y. Senchenkova, S. A. Vital, Z. Al-Yafeai, G. Kaur, E. M. Sparkenbaugh, A. W. Orr, R. Pawlinski, R. P. Hebbel, D. N. Granger, P. Kubes, and F. N. E. Gavins. 2021. 'Targeting the AnxA1/Fpr2/ALX pathway regulates neutrophil function, promoting thromboinflammation resolution in sickle cell disease', Blood , 137: 1538-49.

Bost, P., F. De Sanctis, S. Cane, S. Ugel, K. Donadello, M. Castellucci, D. Eyal, A. Fiore, C. Anselmi, R. M. Barouni, R. Trovato, S. Caligola, A. Lamolinara, M. Iezzi, F. Facciotti, A. Mazzariol, D. Gibellini, P. De Nardo, E. Tacconelli, L. Gottin, E. Polati, B. Schwikowski, I. Amit, and V. Bronte. 2021. 'Deciphering the state of immune silence in fatal COVID-19 patients', Nat Commun, 12: 1428.

Davidson, A. M., J. Wysocki, and D. Batlle. 2020. 'Interaction of SARS-CoV-2 and Other Coronavirus With ACE (Angiotensin-Converting Enzyme)-2 as Their Main Receptor: Therapeutic Implications',Hypertension 
, 76: 1339-49.

De Meyer, S. F., F. Denorme, F. Langhauser, E. Geuss, F. Fluri, and C. Kleinschnitz. 2016. 'Thromboinflammation in Stroke Brain Damage',Stroke, 47: 1165-72.

Diao, B., C. Wang, Y. Tan, X. Chen, Y. Liu, L. Ning, L. Chen, M. Li, Y. Liu, G. Wang, Z. Yuan, Z. Feng, Y. Zhang, Y. Wu, and Y. Chen. 2020. 'Reduction and Functional Exhaustion of T Cells in Patients With Coronavirus Disease 2019 (COVID-19)', Front Immunol, 11: 827.

Gaertner, F., Z. Ahmad, G. Rosenberger, S. Fan, L. Nicolai, B. Busch, G. Yavuz, M. Luckner, H. IshikawaAnkerhold, R. Hennel, A. Benechet, M. Lorenz, S. Chandraratne, I. Schubert, S. Helmer, B. Striednig, K. Stark, M. Janko, R. T. Bottcher, A. Verschoor, C. Leon, C. Gachet, T. Gudermann, Y. Schnitzler M. Mederos, Z. Pincus, M. Iannacone, R. Haas, G. Wanner, K. Lauber, M. Sixt, and S. Massberg. 2017. 'Migrating Platelets Are Mechano-scavengers that Collect and Bundle Bacteria',Cell , 171: 1368-82 e23.

Group, Recovery Collaborative, P. Horby, W. S. Lim, J. R. Emberson, M. Mafham, J. L. Bell, L. Linsell, N. Staplin, C. Brightling, A. Ustianowski, E. Elmahi, B. Prudon, C. Green, T. Felton, D. Chadwick, K. Rege, C. Fegan, L. C. Chappell, S. N. Faust, T. Jaki, K. Jeffery, A. Montgomery, K. Rowan, E. Juszczak, J. K. Baillie, R. Haynes, and M. J. Landray. 2021. 'Dexamethasone in Hospitalized Patients with Covid-19', $N$ Engl J Med, 384: 693-704.

Gu, J., E. Gong, B. Zhang, J. Zheng, Z. Gao, Y. Zhong, W. Zou, J. Zhan, S. Wang, Z. Xie, H. Zhuang, B. Wu, H. Zhong, H. Shao, W. Fang, D. Gao, F. Pei, X. Li, Z. He, D. Xu, X. Shi, V. M. Anderson, and A. S. Leong. 2005. 'Multiple organ infection and the pathogenesis of SARS', J Exp Med , 202: 415-24.

Hoffmann, M., H. Kleine-Weber, S. Schroeder, N. Kruger, T. Herrler, S. Erichsen, T. S. Schiergens, G. Herrler, N. H. Wu, A. Nitsche, M. A. Muller, C. Drosten, and S. Pohlmann. 2020. 'SARS-CoV-2 Cell Entry Depends on ACE2 and TMPRSS2 and Is Blocked by a Clinically Proven Protease Inhibitor', Cell, 181: 271-80 e8.

Holter, J. C., S. E. Pischke, E. de Boer, A. Lind, S. Jenum, A. R. Holten, K. Tonby, A. Barratt-Due, M. Sokolova, C. Schjalm, V. Chaban, A. Kolderup, T. Tran, T. Tollefsrud Gjolberg, L. G. Skeie, L. Hesstvedt, V. Ormasen, B. Fevang, C. Austad, K. E. Muller, C. Fladeby, M. Holberg-Petersen, B. Halvorsen, F. Muller, P. Aukrust, S. Dudman, T. Ueland, J. T. Andersen, F. Lund-Johansen, L. Heggelund, A. M. Dyrhol-Riise, and T. E. Mollnes. 2020. 'Systemic complement activation is associated with respiratory failure in COVID-19 hospitalized patients', Proc Natl Acad Sci U S A , 117: 25018-25.

Kolaczkowska, E., and P. Kubes. 2013. 'Neutrophil recruitment and function in health and inflammation', Nat Rev Immunol , 13: 159-75.

Lam, T. T., N. Jia, Y. W. Zhang, M. H. Shum, J. F. Jiang, H. C. Zhu, Y. G. Tong, Y. X. Shi, X. B. Ni, Y. S. Liao, W. J. Li, B. G. Jiang, W. Wei, T. T. Yuan, K. Zheng, X. M. Cui, J. Li, G. Q. Pei, X. Qiang, W. Y. Cheung, L. F. Li, F. F. Sun, S. Qin, J. C. Huang, G. M. Leung, E. C. Holmes, Y. L. Hu, Y. Guan, and W. C. Cao. 2020. 'Identifying SARS-CoV-2-related coronaviruses in Malayan pangolins', Nature, 583: 282-85.

Lippi, G., M. Plebani, and B. M. Henry. 2020. 'Thrombocytopenia is associated with severe coronavirus disease 2019 (COVID-19) infections: A meta-analysis', Clin Chim Acta , 506: 145-48.

Magro, C., J. J. Mulvey, D. Berlin, G. Nuovo, S. Salvatore, J. Harp, A. Baxter-Stoltzfus, and J. Laurence. 2020. 'Complement associated microvascular injury and thrombosis in the pathogenesis of severe COVID-19 infection: A report of five cases', Transl Res , 220: 1-13.

Maier, C. L., A. D. Truong, S. C. Auld, D. M. Polly, C. L. Tanksley, and A. Duncan. 2020. 'COVID-19associated hyperviscosity: a link between inflammation and thrombophilia?', Lancet, 395: 1758-59.

Manne, B. K., F. Denorme, E. A. Middleton, I. Portier, J. W. Rowley, C. Stubben, A. C. Petrey, N. D. Tolley, L. Guo, M. Cody, A. S. Weyrich, C. C. Yost, M. T. Rondina, and R. A. Campbell. 2020. 'Platelet gene expression and function in patients with COVID-19', Blood , 136: 1317-29. 
McFadyen, J. D., H. Stevens, and K. Peter. 2020. 'The Emerging Threat of (Micro)Thrombosis in COVID-19 and Its Therapeutic Implications', Circ Res , 127: 571-87.

Rapkiewicz, A. V., X. Mai, S. E. Carsons, S. Pittaluga, D. E. Kleiner, J. S. Berger, S. Thomas, N. M. Adler, D. M. Charytan, B. Gasmi, J. S. Hochman, and H. R. Reynolds. 2020. 'Megakaryocytes and platelet-fibrin thrombi characterize multi-organ thrombosis at autopsy in COVID-19: A case series', EClinicalMedicine, 24: 100434 .

Senchenkova, E. Y., J. Ansari, F. Becker, S. A. Vital, Z. Al-Yafeai, E. M. Sparkenbaugh, R. Pawlinski, K. Y. Stokes, J. L. Carroll, A. M. Dragoi, C. X. Qin, R. H. Ritchie, H. Sun, H. H. Cuellar-Saenz, M. R. Rubinstein, Y. W. Han, A. W. Orr, M. Perretti, D. N. Granger, and F. N. E. Gavins. 2019. 'Novel Role for the AnxA1-Fpr2/ALX Signaling Axis as a Key Regulator of Platelet Function to Promote Resolution of Inflammation', Circulation, 140: 319-35.

Senchenkova, E. Y., J. Russell, A. Yildirim, D. N. Granger, and F. N. E. Gavins. 2019. 'Novel Role of T Cells and IL-6 (Interleukin-6) in Angiotensin II-Induced Microvascular Dysfunction', Hypertension , 73: 829-38.

Sinha, P., M. A. Matthay, and C. S. Calfee. 2020. 'Is a "Cytokine Storm" Relevant to COVID-19?', JAMA Intern Med , 180: 1152-54.

Skendros, P., A. Mitsios, A. Chrysanthopoulou, D. C. Mastellos, S. Metallidis, P. Rafailidis, M. Ntinopoulou, E. Sertaridou, V. Tsironidou, C. Tsigalou, M. Tektonidou, T. Konstantinidis, C. Papagoras, I. Mitroulis, G. Germanidis, J. D. Lambris, and K. Ritis. 2020. 'Complement and tissue factor-enriched neutrophil extracellular traps are key drivers in COVID-19 immunothrombosis', J Clin Invest, 130: 6151-57.

Tian, S., Y. Xiong, H. Liu, L. Niu, J. Guo, M. Liao, and S. Y. Xiao. 2020. 'Pathological study of the 2019 novel coronavirus disease (COVID-19) through postmortem core biopsies', Mod Pathol, 33: 1007-14.

Tomar, B., H. J. Anders, J. Desai, and S. R. Mulay. 2020. 'Neutrophils and Neutrophil Extracellular Traps Drive Necroinflammation in COVID-19', Cells , 9.

Veras, F. P., M. C. Pontelli, C. M. Silva, J. E. Toller-Kawahisa, M. de Lima, D. C. Nascimento, A. H. Schneider, D. Caetite, L. A. Tavares, I. M. Paiva, R. Rosales, D. Colon, R. Martins, I. A. Castro, G. M. Almeida, M. I. F. Lopes, M. N. Benatti, L. P. Bonjorno, M. C. Giannini, R. Luppino-Assad, S. L. Almeida, F. Vilar, R. Santana, V. R. Bollela, M. Auxiliadora-Martins, M. Borges, C. H. Miranda, A. Pazin-Filho, L. L. P. da Silva, L. D. Cunha, D. S. Zamboni, F. Dal-Pizzol, L. O. Leiria, L. Siyuan, S. Batah, A. Fabro, T. Mauad, M. Dolhnikoff, A. Duarte-Neto, P. Saldiva, T. M. Cunha, J. C. Alves-Filho, E. Arruda, P. Louzada-Junior, R. D. Oliveira, and F. Q. Cunha. 2020. 'SARS-CoV-2-triggered neutrophil extracellular traps mediate COVID-19 pathology', J Exp Med, 217.

Vital, S. A., F. Becker, P. M. Holloway, J. Russell, M. Perretti, D. N. Granger, and F. N. Gavins. 2016. 'Formyl-Peptide Receptor 2/3/Lipoxin A4 Receptor Regulates Neutrophil-Platelet Aggregation and Attenuates Cerebral Inflammation: Impact for Therapy in Cardiovascular Disease', Circulation, 133: 2169-79.

Yang, J., Y. Zheng, X. Gou, K. Pu, Z. Chen, Q. Guo, R. Ji, H. Wang, Y. Wang, and Y. Zhou. 2020. 'Prevalence of comorbidities and its effects in patients infected with SARS-CoV-2: a systematic review and meta-analysis', Int J Infect Dis, 94: 91-95.

Yao, D., K. Yan, J. Duan, X. Zhang, and L. Zhou. 2020. 'Coronavirus Disease 2019 (COVID-19): Prevention and Control in Gynecological Outpatient Clinic', Front Public Health , 8: 618494.

Zhu, Z., X. Lian, X. Su, W. Wu, G. A. Marraro, and Y. Zeng. 2020. 'From SARS and MERS to COVID-19: a brief summary and comparison of severe acute respiratory infections caused by three highly pathogenic human coronaviruses', Respir Res, 21: 224.

Zuo, Y., S. Yalavarthi, H. Shi, K. Gockman, M. Zuo, J. A. Madison, C. Blair, A. Weber, B. J. Barnes, M. Egeblad, R. J. Woods, Y. Kanthi, and J. S. Knight. 2020. 'Neutrophil extracellular traps in COVID-19',JCI 
Insight , 5 .

Figure Legend

\section{Figure 1: Thromboinflammation in COVID-19}

(1) SARS-CoV-2 binds via the human angiotensin-converting enzyme 2 (ACE-2) receptor expressed on the endothelial surface. The virus activates monocytes releasing a cytokine storm (including interleukin [IL]-6, IL-1 $\beta$, IL-2, IL-6, IL-7, IL-8, IL-10, IL-17, TNF- $\alpha$, chemokine ligand-2 (CCL2), CCL3, interferon gamma-inducible protein, C-X-C motif chemokine ligand-10, and monocyte chemoattractant protein-1, all of which correlate with adverse clinical outcomes.(2) The inflammatory effects of cytokines also activate vascular endothelial cells, disrupting endothelial function and integrity, which leads to increased platelet and neutrophil recruitment.(3) Increased neutrophil at inflammatory site results in increased expression of adhesion molecules such as L-selectin, P-selectin, intracellular adhesion molecule-1, on endothelial cells. Activated neutrophils release their neutrophil extracellular traps (NETs, which contain a backbone of DNA and citrullinated histone [H3Cit], cathepsin $\mathrm{G}$, and neutrophil elastase [NE]) which trap platelets, increasing their activation and aggregation, this in turn further activates neutrophils to produce more NETs. (4) This cycle of events triggers coagulation cascade activation which in turn increases fibrin formation and thrombosis.

\section{Hosted file}

Figure 1 .pptx available at https://authorea.com/users/408561/articles/518514thromboinflammation-in-covid-19-the-clot-thickens 